\title{
Efficacy of a transdiagnostic ecological momentary intervention for improving self- esteem (SELFIE) in youth exposed to childhood adversity: study protocol for a multi-center randomized controlled trial
}

Maud Daemen ${ }^{1,2}$ (D) Mary Rose Postma ${ }^{1,3}$, Ramon Lindauer ${ }^{4,5}$, Iris Hoes-van der Meulen ${ }^{6}$, Dorien Nieman ${ }^{7}$, Philippe Delespaul ${ }^{1}$, Josefien Johanna Froukje Breedvelt ${ }^{7,8}$, Mark van der Gaag ${ }^{9,10}$, Wolfgang Viechtbauer ${ }^{1}$, Koen Schruers ${ }^{1,11}$, David van den Berg ${ }^{9,10}$, Claudi Bockting ${ }^{7,8}$, Therese van Amelsvoort ${ }^{1,3}$ and Ulrich Reininghaus ${ }^{1,2,12^{*}}$

\begin{abstract}
Background: Targeting low self-esteem in youth exposed to childhood adversity is a promising strategy for preventing adult mental disorder, but psychological help remains difficult to access and accept for youth, calling for novel, youth-friendly approaches. Mobile Health (mHealth) and, most prominently, ecological momentary interventions (EMIs) provide a unique opportunity to deliver youth-friendly, personalized, real-time, guided self-help interventions. The aim of this study is to investigate the efficacy of a novel, accessible, transdiagnostic ecological momentary intervention for improving self-esteem ('SELFIE') in youth with prior exposure to childhood adversity.

Methods/design: In a parallel-group, assessor-blind, multi-center randomized controlled trial, individuals aged 1226 years with prior exposure to childhood adversity and low self-esteem will be randomly allocated to SELFIE in addition to treatment as usual (TAU) as the experimental condition or the control condition of TAU only, which will include access to all standard health care. SELFIE is a digital guided self-help intervention administered through a smartphone-based app to allow for interactive, personalized, real-time and real-world transfer of intervention components in individuals' daily lives, blended with three training sessions delivered by trained mental health professionals over a 6-week period. Outcomes will be assessed at baseline, post-intervention, and 6-month followup by blinded assessors. The primary outcome will be the level of self-esteem as measured with the Rosenberg Self-Esteem Scale (RSES).
\end{abstract}

\footnotetext{
* Correspondence: ulrich.reininghaus@zi-mannheim.de

'Department of Psychiatry and Neuropsychology, School for Mental Health and Neuroscience, Maastricht University, Maastricht, The Netherlands

${ }^{2}$ Department of Public Mental Health, Central Institute of Mental Health, Medical Faculty Mannheim, University of Heidelberg, Mannheim, Germany

Full list of author information is available at the end of the article
}

(c) The Author(s). 2021 Open Access This article is licensed under a Creative Commons Attribution 4.0 International License, which permits use, sharing, adaptation, distribution and reproduction in any medium or format, as long as you give appropriate credit to the original author(s) and the source, provide a link to the Creative Commons licence, and indicate if changes were made. The images or other third party material in this article are included in the article's Creative Commons licence, unless indicated otherwise in a credit line to the material. If material is not included in the article's Creative Commons licence and your intended use is not permitted by statutory regulation or exceeds the permitted use, you will need to obtain permission directly from the copyright holder. To view a copy of this licence, visit http://creativecommons.org/licenses/by/4.0/ The Creative Commons Public Domain Dedication waiver (http://creativecommons.org/publicdomain/zero/1.0/) applies to the data made available in this article, unless otherwise stated in a credit line to the data. 
Discussion: The current study is the first to establish the efficacy of an EMI focusing on improving self-esteem transdiagnostically in youth exposed to childhood adversity. If this trial provides evidence on the efficacy of SELFIE, it has significant potential to contribute to minimizing the deleterious impact of childhood adversity and, thereby, preventing the development of mental disorder later in life.

Trial registration: Netherlands Trial Register NL7129 (NTR7475). Registered on 9 November 2018

Keywords: SELFIE, Ecological momentary intervention, mHealth, Experience Sampling Method, Self-esteem, Childhood trauma, Childhood adversity

\section{Introduction}

The majority of mental disorders first emerge in youth and, as such, contribute substantially to disease burden, which is higher in youth than during any other developmental period [1-5]. More specifically, $50 \%$ of lifetime cases of mental disorder have started by age 14 years and three quarters by age $24[2,6]$. Mental disorders in youth aged 10-24 years are associated with an immense cost [7-9] and have been found to be the leading cause of disease burden in high-income countries $[4,5]$. Onset of a mental disorder may disrupt critical age-specific developmental, interpersonal, occupational, and educational milestones [10-12] and indicates a need for close scrutiny of the complex interplay between risk and protective factors in childhood and adolescence. Recently, transdiagnostic frameworks have become more prominent (e.g., the Hierarchical Taxonomy of Psychopathology (HiTOP) [13, 14], which broadly posit that symptoms of psychopathology are transdiagnostic in the early stages [15] and might result in a wide range of psychopathology later in life [12, 16]. Furthermore, during the ongoing COVID-19 pandemic, measures to control SARS-CoV-2 transmission rates have been shown to have negative effects on mental health, especially in youth [17-19]. All this highlights the value of transdiagnostic preventive interventions to improve well-being and resilience in youth and prevent morbidity later in life in order to reduce burden for individuals, families, and the wider society $[2,11,20-22]$.

Youth referred to mental health services have experienced disproportionate levels of childhood adversity (i.e., abuse, neglect, bullying and household discord) [23-31], which is one of the most pervasive risk factors for developing a range of mental disorders [25, 32, 33]. For example, in a nation-wide Dutch study of help-seeking adolescents and young adults with an Ultra High Risk state for Psychosis (UHR), a high prevalence was found for physical (20.9\%), sexual (24.8\%), and emotional (46.7\%) abuse, as well as physical (41.9\%) and emotional (66.7\%) neglect [29]. Also, in a study based on a representative sample drawn randomly from the general population in the Netherlands, it was shown that $29.7 \%$ experienced one or more adversities during their childhood [34]. Current estimates of attributable risks further suggest that interventions targeted at averting childhood adversity from exerting its adverse effects can prevent a substantial proportion of the incidence of adult mental disorder, and, thereby, have a sizeable public health impact and reduce societal costs [26, 35]. While primary prevention of childhood adversity through universal, population-based strategies is of prime importance, it remains difficult to achieve for all, and, hence, interventions targeting the negative psychological consequences of childhood adversity in youth are a promising selective prevention strategy for adverse outcomes later in life with tangible public health implications [31, 36].

One important psychological mechanism in pathways from childhood adversity to adult psychopathology is low self-esteem [37, 38]. Youth is a critical period for the development of self-esteem. Self-esteem is essential to well-being and mental health per se, with a substantial impact on the development and maintenance of severe mental disorders [39]. There is now substantial evidence to suggest that exposure to childhood adversity has detrimental effects on self-esteem [40-43]. The current evidence further suggests that childhood adversity exerts its detrimental effects on risk of later psychopathology precisely via pathways through low selfesteem [36, 37, 44-47]. The prevalence of low selfesteem in help-seeking youth has been reported to be around 45\% [48]. Taken together, targeting low selfesteem at an early stage in youth exposed to childhood adversity is a promising strategy for preventing mental disorder and reducing societal costs.

Current psychological help, including prevention, however, remains difficult to access and accept for youth and has limited efficacy under real-world conditions, calling for novel approaches $[49,50]$. While conventional interventions have proven efficacious in reducing psychiatric symptoms via enhancing self-esteem [51], a key next step is to develop and evaluate interventions that are specifically geared toward the specific needs of youth. This is what the current study is designed to achieve. The recent advances in information and communication technologies have led to the development of 
mobile Health (mHealth) interventions and, most prominently, ecological momentary interventions (EMIs) [5256]. EMIs provide a unique opportunity to deliver youth-friendly, accessible, personalized, real-time, guided self-help interventions targeting candidate psychological mechanisms in daily life and thereby prevent mental disorder and reduce disease burden. This enables youth to access interventions that are individually adapted to their needs in a given moment and context (e.g., by offering interventions specifically tailored for helping participants in moments of low self-esteem). Recently, the term "Just-In-Time Adaptive Interventions (JITAIs)" has been started to be used by some authors $[57,58]$, positing that novel characteristics of JITAIs are that interventions are initiated by push notifications and dynamically initiated by the app. However, these features have been part of EMIs from the outset, and, hence, if anything JITAIs may be used synonymously with EMIs, which have been proposed at a much earlier point. EMIs are ideally placed for enhancing access to mental health services for youth depending on their needs and preferences by delivering low-threshold interventions by mental health professionals as one component that can be rolled out across child, adolescent and adult mental health services.

Previous studies of conventional interventions suggest that psychiatric symptoms, such as anxiety and depression symptoms, may be reduced through enhancing selfesteem [51]. However, these interventions are not tailored toward the specific preferences and needs of youth as naturally occurring in daily life. While EMIs such as the SELFIE intervention provide a unique opportunity to deliver youth-friendly, accessible, personalized, real-time interventions in daily life, robust trial-based evidence on EMIs and other mHealth interventions remains very limited [22, 52, 53, 59-62].

The overall aim of the current study is to test the efficacy of a novel, accessible, transdiagnostic ecological momentary intervention (EMI) for improving selfesteem ("SELFIE") in youth aged 12-26 with prior exposure to childhood adversity in a multi-center randomized controlled trial (RCT). The SELFIE intervention will be administered in addition to treatment as usual (TAU) (experimental condition) and compared to a control condition of TAU only, which will include (access to) standard health care.

The specific objectives of this study are to:

1) Test the efficacy of the SELFIE intervention on improving self-esteem at post-intervention and 6month follow-up (primary outcome);

2) Test the efficacy of the SELFIE intervention on improving momentary self-esteem, positive and negative schematic beliefs of self, resilience, emotional well-being, general psychopathology, functioning, and quality of life at post-intervention and 6-month follow-up (secondary outcomes);

3) Establish whether the effects of the SELFIE intervention on primary and secondary outcomes hold at 18-month and 24-month follow-up;

4) Examine the cost effectiveness and cost utility of the SELFIE intervention;

5) Assess the acceptability, safety, adherence and fidelity of the SELFIE intervention.

\section{Methods \\ Study design}

In a two-arm parallel-group, assessor-blind multicenter randomized controlled trial, individuals aged between 12 and 26 years with prior exposure to childhood adversity and low self-esteem will be randomly allocated to SELFIE in addition to TAU as the experimental condition or a control condition of TAU only, which includes (access to) standard health care and social services. Participants will be recruited from mental health services in Noord-Holland, ZuidHolland, and Limburg (the Netherlands) and from the general population (e.g., via social media). Outcomes will be measured at baseline (i.e., before randomization), post-intervention (i.e., after the 6week intervention period), and 6-month, 18-month, and 24-month follow-up (i.e., 6, 18 and 24 months after completing the intervention period) by blind assessors (see Figs. 1 and 2). Randomization will be conducted independently of the research team through a computer-generated sequence, stratified by region of collaborating centers or as external admission. All outcomes will be measured and the statistical analysis will be performed blind to treatment allocation.

\section{Participants}

A sample of 174 individuals aged 12-26 with prior exposure to childhood adversity and low self-esteem will be recruited. Participants will be recruited from collaborating mental health services in three regions in the Netherlands: Noord-Holland (Amsterdam University Medical Centers (Location AMC); Levvel), Zuid-Holland (Parnassia Group; Prodeba), and Limburg (Mondriaan; Lionarons GGZ; Koraalgroep). In addition, participants from the general population, who do not seek help from collaborating mental health services, will be recruited, e.g., via (targeted adverts on) social media, schools, social services, and flyers at relevant public locations. All individuals presenting to collaborating mental health services will be approached by their treating clinician, who will provide initial information about the study. If the individual is interested in the study, their treating clinician will, in agreement with the potential participant, pass on 


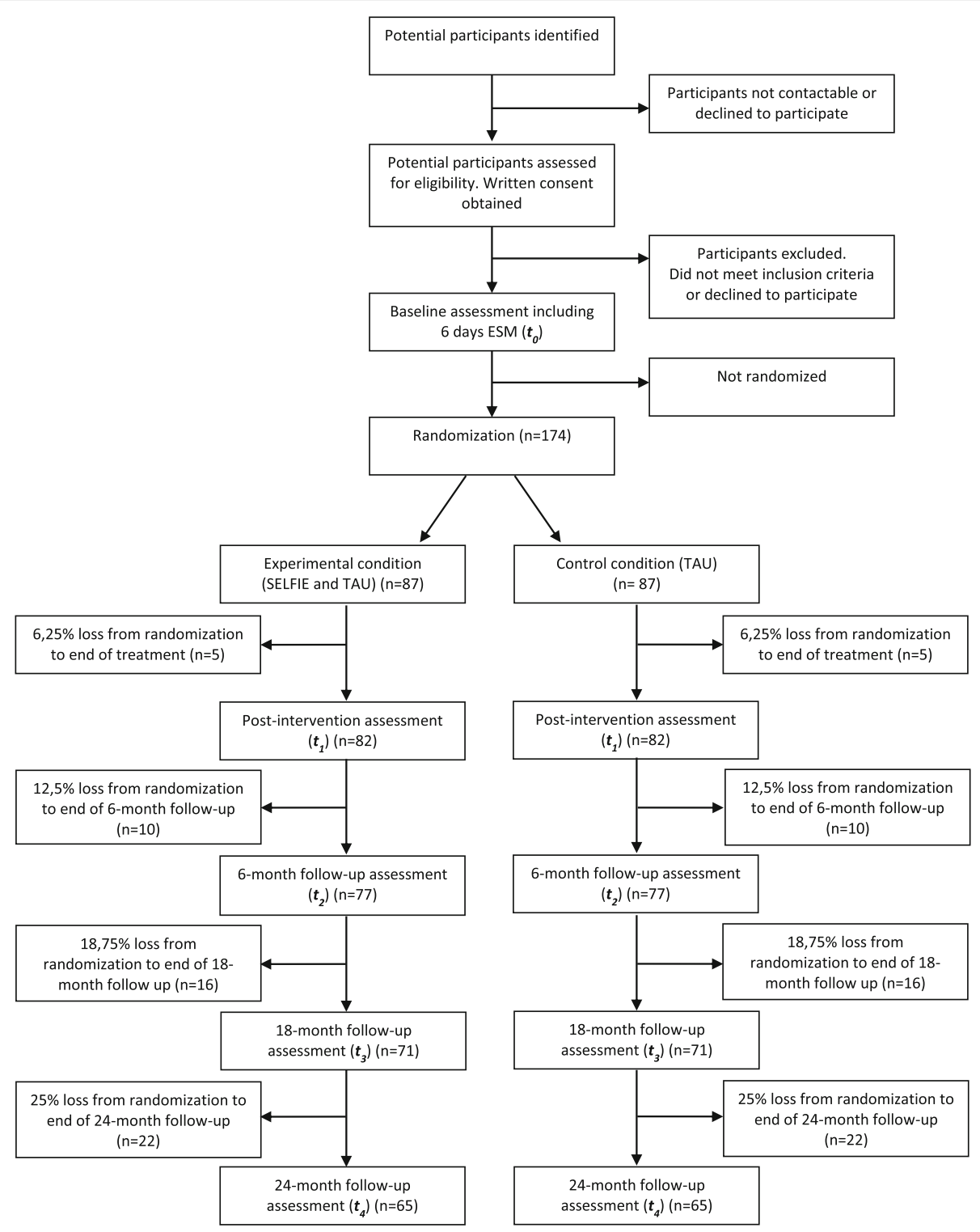

Fig. 1 Overview of the study design

their contact details to the research team. All potential participants (including those recruited via social media, etc.) will be contacted by the research team and will be fully informed about the study. One week later, informed consent will be obtained (if applicable, also from parents/legal guardians), which can be withdrawn by participants at any time and without having any negative consequences for their access to standard health care). For participants under the age of 16, both parents (or the legal guardian) and participants will receive detailed information about the intervention. In addition, parents (or the legal guardian) and the researcher will consider possible negative reactions of the underage participant to the intervention procedure. Further, it will be determined together with the parents (or the legal guardian) prior to the intervention what the researcher will do in case of reluctance of the underage participant and which behavior of the participant commonly reflects reluctance. It will be discussed with the parents (or the legal guardian) when the study should be stopped in case of reluctance of their child.

Potential participants will then be asked to complete the screening questionnaires to assess whether they meet the inclusion criteria. Participants aged 16 years or older will be financially compensated for their time, and travel expenses will be fully reimbursed. To minimize loss to follow-up, researchers maintain contact with participants on a regular basis. Also, participants will receive a small additional financial reimbursement for completing all follow-up assessments. 


\begin{tabular}{|c|c|c|c|c|c|c|c|}
\hline \multirow[b]{3}{*}{ TIMEPOINT** } & \multirow{3}{*}{$\begin{array}{l}\text { Enrolment } \\
-t_{1}\end{array}$} & \multirow[b]{3}{*}{\begin{tabular}{|l}
$t_{0}$ \\
Bassline \\
\end{tabular}} & \multicolumn{5}{|c|}{ STUDY PERIOD } \\
\hline & & & \multirow[t]{2}{*}{ Allocation } & \multicolumn{4}{|c|}{\begin{tabular}{|l|} 
Post-allocation \\
\end{tabular}} \\
\hline & & & & $\begin{array}{l}t_{1} \\
\text { post- } \\
\text { intervention } \\
\end{array}$ & $\begin{array}{ll}t_{2} \\
6-\text { month } \\
\text { follow-up } \\
\end{array}$ & \begin{tabular}{|l|l}
$t_{3}$ \\
18-month \\
follow-up \\
\end{tabular} & \begin{tabular}{|l|}
$t_{4}$ \\
24-month \\
follow-up \\
\end{tabular} \\
\hline \multicolumn{8}{|l|}{ ENROLMENT: } \\
\hline Informed consent & $x$ & & & & & & \\
\hline \multicolumn{8}{|l|}{ Childhood Trauma Questionnaire (СтQ) } \\
\hline Parental Conflict (CECA) & $x$ & & & & & & \\
\hline Retrospective Bullying Questionnaire (RBQ) & $x$ & & & & & & \\
\hline Rosenberg Self-Esteem Scale (RSES) & $x$ & & & & & & \\
\hline Screening Questionnaire & $x$ & & & & & & \\
\hline Allocation & & & $x$ & & & & \\
\hline \multicolumn{8}{|l|}{ INTERVENTIONS: } \\
\hline \multicolumn{8}{|l|}{ Experimental condition (SELFIE + TAU) } \\
\hline Control condition (TAU) & & & & & & & \\
\hline \multicolumn{8}{|l|}{$\begin{array}{ll}\text { ASSESSMENTS: } & \text { Outcome measures }\end{array}$} \\
\hline $\begin{array}{l}\text { Ecological Momentary Assessment (EMA) } \\
\text { questionnaire }\end{array}$ & & $x$ & & $x$ & $x$ & $x$ & $x$ \\
\hline EMA briefing questionnaire & & $x$ & & $x$ & $x$ & $x$ & $x$ \\
\hline EMA debriefing questionnaire & & $x$ & & $x$ & $x$ & $x$ & $x$ \\
\hline Self-Esteem Rating Scale (SERS) & & $x$ & & $x$ & $x$ & $x$ & $x$ \\
\hline Rosenberg Self-Esteem Scale (RSES) & & $x$ & & $x$ & $x$ & $x$ & $x$ \\
\hline Brief Core Schema Scales (BCSS) & & $x$ & & $x$ & $x$ & $x$ & $x$ \\
\hline $\begin{array}{l}\text { Temperament and Characteristic Inventory } \\
\text { (TCI) }\end{array}$ & & $x$ & & & & & \\
\hline Kessler Psychological Distress Scale (K10) & & $x$ & & $x$ & $x$ & $x$ & $x$ \\
\hline Symptom Checklist (SCL-90-R) & & $x$ & & $x$ & $x$ & $x$ & $x$ \\
\hline Positive and Negative Affect Scale (PANAS) & & $x$ & & $x$ & $x$ & $x$ & $x$ \\
\hline $\begin{array}{l}\text { Social and Occupational Functioning } \\
\text { Assessment Scale (SOFAS) }\end{array}$ & & $x$ & & $x$ & $x$ & $x$ & $x$ \\
\hline Global Assessment of Functioning (GAF) & & $x$ & & $x$ & $x$ & $x$ & $x$ \\
\hline $\begin{array}{l}\text { World Health Organisation Quality of Life } \\
\text { Instrument-Brief (WHOQOL-BREF) }\end{array}$ & & $x$ & & $x$ & $x$ & $x$ & $x$ \\
\hline Brief Psychiatric Rating Scale (BPRS) & & $x$ & & $x$ & $x$ & $x$ & $x$ \\
\hline $\begin{array}{l}\text { Trimbos Institute and Institute of Medical } \\
\text { Technology Assessment Questionnaire for } \\
\text { Costs associated with Psychiatric IIness (TiC- } \\
\text { P) }\end{array}$ & & $x$ & & $x$ & $x$ & $x$ & $x$ \\
\hline $\begin{array}{l}\text { EQ-5D 3-level version of the 'EuroQol' group } \\
\text { (EQ-5D-5L) }\end{array}$ & & $x$ & & $x$ & $x$ & $x$ & $x$ \\
\hline \multicolumn{8}{|l|}{ Other study parameters } \\
\hline Socio-demographic schedule & & $x$ & & & & & \\
\hline $\begin{array}{l}\text { Composite International Diagnostic } \\
\text { Interview (CIDI-B-J-L) }\end{array}$ & & $x$ & & & & & \\
\hline Medication use & & $x$ & & $x$ & $x$ & $x$ & $x$ \\
\hline Treatment Classification (present and past) & & $x$ & & $x$ & $x$ & $x$ & $x$ \\
\hline CECA (social support) & & $x$ & & & & & \\
\hline Working Alliance Inventory (WAI) & & & & $\mathrm{x}$ & & & \\
\hline $\begin{array}{l}\text { Debriefing questionnaire: SELFIE } \\
\text { Intervention }\end{array}$ & & & & $x$ & & & \\
\hline Debriefing questionnaire: SELFIE Follow-Up & & & & & $x$ & $x$ & $x$ \\
\hline Qualitative interview (process evaluation) & & & & $\mathrm{x}$ & & & \\
\hline
\end{tabular}

Fig. 2 Standard Protocol Items: Recommendations for Interventional Trial (SPIRIT) figure. Ecological momentary intervention for improving selfesteem (SELFIE): schedule of enrolment, interventions, and assessments 


\section{Inclusion criteria}

Inclusion criteria are as follows (see Table 1 for more detail): (1) aged between 12 and 26 years, (2) exposure to childhood adversity (physical, sexual, or emotional abuse, emotional or physical neglect, peer bullying, or parental conflict), (3) self-esteem below average measured with the Rosenberg Self-Esteem Scale (RSES) [51, 68, 69], (4) willingness to participate, (5) ability to give informed consent, and (6) parental consent for minors.

\section{Exclusion criteria}

Subjects will be excluded if their command of Dutch is insufficient or if their psychiatric symptoms are due to an organic cause.

\section{Intervention}

\section{Control condition: treatment as usual (TAU)}

Participants allocated to the control condition will receive treatment as usual (TAU), which will include access to all standard health care and social services. Specifically, this will include all the input from their general practitioner and other providers of health and social services that they would receive if they did not participate in the study, except for manualized treatment that explicitly addresses self-esteem as primary target (e.g., COMET or EMDR $[51,70,71])$ during the intervention period.

\section{Table 1 Eligibility criteria}

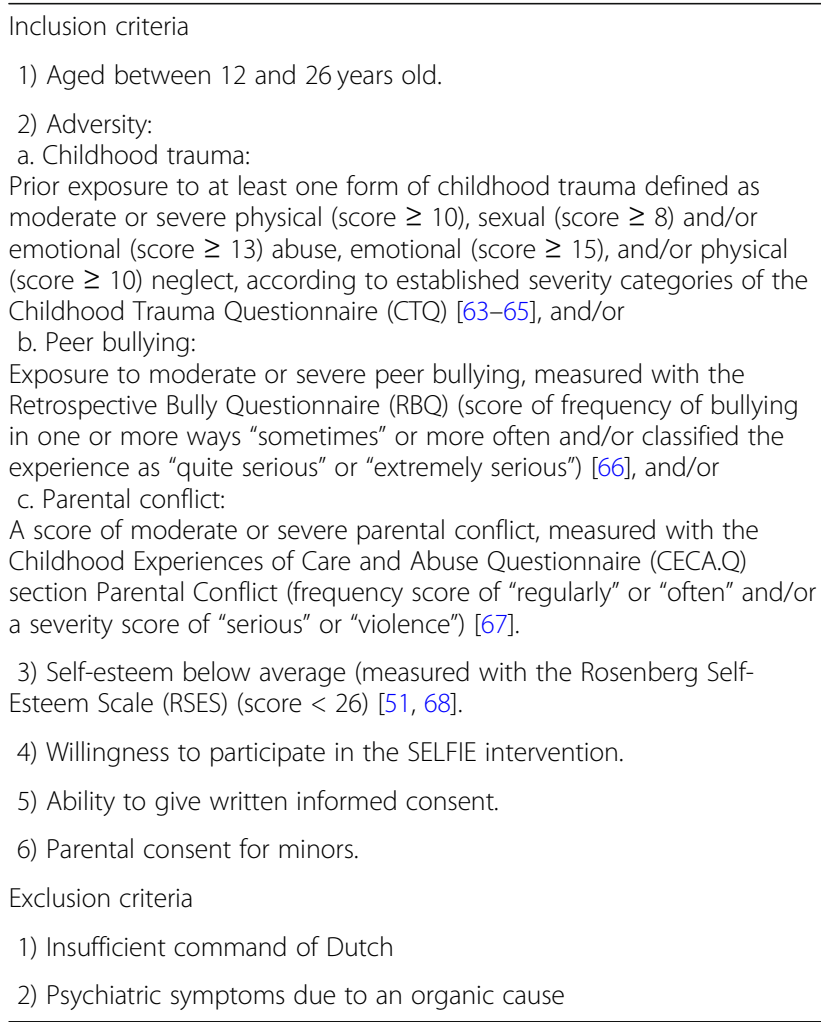

\section{Experimental condition: SELFIE + TAU}

Participants allocated to the experimental condition will receive the manualized SELFIE intervention within a 6week period in addition to TAU. The intervention consists of three face-to-face sessions, each for around 60 min, delivered by SELFIE therapists, who will be trained mental health professionals (e.g., psychologists, social workers and mental health nurse specialists trained in the SELFIE intervention and receiving regular supervision and inter-vision led by a clinical psychologist), three e-mail contacts, and an EMI administered through a smartphone-based app (i.e., the PsyMate ${ }^{\bullet}$ app) for adaptive real-time and real-world transfer of intervention components tailored to person, moment, and context, delivered over a 6-week intervention period. Due to the COVID-19 pandemic, some of the face-to-face sessions will be offered through a secure and encrypted video conferencing system. The intervention is based on principles of EMIs [22, 52-56, 62, 71], and a guided self-help approach using principles of cognitive-behavioral therapy (CBT), aimed at modifying cognitive bias inherent to negative self-esteem and developing and practicing a new behavioral repertoire guided by therapists using modeling and shaping as additional important therapeutic techniques $[72,73]$. Delivering the intervention in individuals' daily lives, and enabling youth to benefit from this intervention in a given moment and context, when most needed (e.g., in moments of low self-esteem) is the key goal of the 6-week SELFIE intervention. Therefore, in the first introductory session, participants will either receive a study smartphone with the app already installed or will be asked to install it on their own smartphone by the SELFIE therapist, who will explain the SELFIE intervention in detail and ask the participant to complete examples of training tasks on the app in order to address the self-selected goals the participant wants to work on in the 6-week intervention period. The app will offer participants "enhancing," "consolidating," and "interactive" tasks (see Table 2) [72, 73]. In enhancing tasks, new intervention components will be introduced and practiced, some of which will be modified and extended over the study period. Consolidating tasks will ask participants to practice previously learned components of enhancing tasks on a daily basis. For these tasks, participants will be reminded by the app between 1 and 3 times per day (varying by intervention week). During the intervention period, the Experience Sampling Method (ESM), a structured dairy technique, will be used to assess momentary self-esteem, affect, and pleasantness of activities and events, six times a day, on days 3,4 , and 5 in each of the six intervention weeks using a time-based design with stratified random sampling (i.e., with ESM assessments scheduled at random within set blocks of time) to allow for interactive tasks. 
Table 2 Key components of the SELFIE intervention

\begin{tabular}{|c|c|c|c|c|c|c|}
\hline & 1 & 2 & 3 & 4 & 5 & 6 \\
\hline $\begin{array}{l}\text { Training } \\
\text { session }\end{array}$ & $\begin{array}{l}\text { Face-to-face } \\
\text { session } 1\end{array}$ & E-mail contact 1 & Face-to-face session 2 & E-mail contact 2 & $\begin{array}{l}\text { Face-to-face session } \\
3\end{array}$ & $\begin{array}{l}\text { E-mail } \\
\text { contact } 3\end{array}$ \\
\hline $\begin{array}{l}\text { Enhancing } \\
\text { EMI tasks }\end{array}$ & $\begin{array}{l}\text { Formulating a } \\
\text { new positive core } \\
\text { belief } \\
+ \\
+ \\
\text { Positive datalog } \\
\text { (enter daily } \\
\text { successes) }\end{array}$ & $\begin{array}{l}\text { Personal positive } \\
\text { qualities (integrated } \\
\text { in positive datalog) } \\
+ \\
\text { Tips to identify more } \\
\text { positive qualities } \\
+ \\
\text { One-minute exercise } \\
\text { (listing (previously } \\
\text { identified) positive } \\
\text { qualities) }\end{array}$ & $\begin{array}{l}\text { Overview old } \\
\text { behavioral patterns } \\
+ \\
\text { Development of new } \\
\text { behavior patterns }\end{array}$ & $\begin{array}{l}\text { Expanding the positive } \\
\text { datalog with successes } \\
\text { arising from new } \\
\text { behavioral patterns }\end{array}$ & $\begin{array}{l}\text { Strategies to deal } \\
\text { with criticism } \\
+ \\
\text { A critical look at } \\
\text { criticism } \\
+ \\
\text { Cost-benefit analysis } \\
\text { of perfectionism } \\
+ \\
\text { The minimum } \\
\text { programme } \\
\text { (practicing to } \\
\text { perform less than } \\
\text { perfect) }\end{array}$ & $\begin{array}{l}\text { Writing a } \\
\text { positive story } \\
\text { about } \\
\text { yourself } \\
+ \\
\text { Maintenance } \\
\text { plan (for after } \\
\text { the } \\
\text { intervention) }\end{array}$ \\
\hline $\begin{array}{l}\text { Consolidating } \\
\text { EMI tasks }\end{array}$ & $\begin{array}{l}\text { Positive datalog } \\
+ \\
\text { Tips to add more } \\
\text { successes in the } \\
\text { positive datalog } \\
+ \\
\text { Rating credibility } \\
\text { of the new core } \\
\text { belief }\end{array}$ & $\begin{array}{l}\text { Positive datalog } \\
+ \\
\text { Tips positive datalog } \\
+ \\
\text { One-minute exercise } \\
+ \\
\text { Rating credibility of } \\
\text { the new core belief }\end{array}$ & $\begin{array}{l}\text { Positive datalog } \\
+ \\
\text { One-minute exercise } \\
+ \\
\text { Rating credibility of } \\
\text { the new core belief }\end{array}$ & $\begin{array}{l}\text { Positive datalog } \\
+ \\
\text { One-minute exercise } \\
+ \\
\text { Expanding new behavior } \\
\text { patterns } \\
+ \\
\text { Rating credibility of the } \\
\text { new core belief }\end{array}$ & $\begin{array}{l}\text { Positive datalog } \\
+ \\
\text { One-minute exercise } \\
+ \\
\text { A critical look at } \\
\text { criticism } \\
+ \\
\text { Rating credibility of } \\
\text { the new core belief }\end{array}$ & $\begin{array}{l}\text { Positive } \\
\text { datalog } \\
+ \\
\text { One-minute } \\
\text { exercise } \\
+ \\
\text { Rating } \\
\text { credibility of } \\
\text { the new core } \\
\text { belief }\end{array}$ \\
\hline $\begin{array}{l}\text { Interactive } \\
\text { EMI tasks }\end{array}$ & $\begin{array}{l}\text { Positive datalog } \\
\text { (adding } \\
\text { successes) } \\
\text { Or } \\
\text { Positive datalog } \\
\text { (viewing } \\
\text { previously } \\
\text { identified } \\
\text { successes) }\end{array}$ & & $\begin{array}{l}\text { Positive datalog } \\
\text { (adding successes } \\
\text { and/or positive } \\
\text { qualities) } \\
\text { Or } \\
\text { Positive datalog } \\
\text { (viewing previously } \\
\text { identified successes } \\
\text { and qualities) }\end{array}$ & & $\begin{array}{l}\text { A critical look at } \\
\text { criticism }\end{array}$ & \\
\hline
\end{tabular}

See Postma [73] and De Neef [72] for more details

Interactive tasks will be provided based on their ESM ratings of (positive and negative) affect, momentary selfesteem and pleasantness of activities and events. For example (in week 1), participants will be provided with an interactive task, offering them to add more successes to their positive datalog when they scored high on positive affect, momentary self-esteem and/or pleasantness of activities. Participants can discontinue the intervention at any time upon request without negative consequences.

\section{Outcome measures}

After obtaining written informed consent and eligibility assessment, participants will complete a range of selfreport, interview-based and computer-based measures to assess primary and secondary outcomes and other study parameters. Participants will complete self-report questionnaires using a smartphone-based app (i.e., the PsyMate ${ }^{\bullet}$ app). Interviews will be conducted using a secure and encrypted video conferencing system. In addition, ESM data will be collected following the protocol from previous ESM studies using the PsyMate ${ }^{\bullet}$ app to measure momentary self-esteem, emotional well-being, stress sensitivity, threat anticipation, and psychotic experiences in daily life for a period of 6 consecutive days [22, 31, 62, 74-76]. On each day, participants will be asked eight times per day to complete an ESM, which will be scheduled at random within set blocks of time. At the end of the 6-day baseline ESM period, participants will be asked to complete a short debriefing questionnaire. All the above-mentioned measures will be assessed at baseline (i.e., before randomization), postintervention (i.e., after the 6-week intervention period), and 6-month follow-up. Please see Fig. 2 (SPIRIT Figure) for details of assessment at each time point. All assessments will be checked for quality and completeness by another member of the research team and an extensive data checking and cleaning will be adhered to as a quality control measure.

\section{Primary outcome}

The primary outcome will be global self-esteem, measured with the Rosenberg Self-Esteem Scale (RSES) [69], 
which is a widely used measure to assess global selfesteem with good reliability and validity $[68,77]$. The RSES consists of ten items rated on a 4-point Likert scale ranging from "strongly agree" to "strongly disagree". The level of global self-esteem, operationalized as the total score of the RSES, will be compared between the experimental and the control condition at postintervention and 6-month follow-up (H1).

\section{Secondary outcomes}

Secondary outcomes will include the level of momentary, positive and negative self-esteem, resilience, emotional well-being, positive and negative schematic beliefs of self, psychological distress, functioning, subjective quality of life, general psychopathology, clinical symptoms and health-related quality of life, service use (including admission to inpatient services) and cost, which will be compared between the experimental and control condition at post-intervention and at 6-month follow-up (H2). In addition, all secondary outcomes (incl. levels of global self-esteem, operationalized using the total score of the RSES (see previous section)) will be compared between the experimental and control condition and at 18and 24-month follow-up (H3).

Momentary self-esteem will be assessed with four items, rated on a 7-point scale, using the $\operatorname{ESM}[78,79]$. The mean score will be used for analysis. Positive and negative self-esteem will be measured with the SERS, which is a 20-item rating scale to assess these two dimensions of self-esteem separately with good reliability and validity [80]. The total sum score of the positive dimension and the total sum score of the negative dimension will be used in the analysis. Momentary resilience will be assessed with the ESM positive affective recovery from event-related stress in daily life (operationalized as the return to baseline levels of positive affect following event-related stress) $[31,74,76,81]$. We will assess emotional well-being using the Positive and Negative Affect Scale (PANAS) [82] based on the total sum score of the negative affect items and the total sum score of the positive affect items. Also, a 5-item ESM measure will be used for assessing negative affect and a 4-item ESM measure of positive affect [31, 74, 83]. For both measures, a mean score will be used in the analysis. The Brief Core Schema Scale (BCSS) will be used as an established measure of positive and negative schematic beliefs of self and others [84]. The following four total scores (all consisting of six items) will be obtained for use in the analysis: negative-self, positive-self, negativeothers, and positive-others. Psychological distress will be measured with the Kessler Psychological Distress Scale (K10), which is widely used and well-validated in youth $[85,86]$. A total sum score ranging from 10 to 50 will be used for analysis.
The Social and Occupational Functioning Assessment Scale (SOFAS) [87] and the Global Assessment of Functioning (GAF) scale [88] will be used as a well-validated measure of functioning in youth [86]. The overall level of functioning rated by researchers on a scale of 0 to 100 will be used in the analysis.

Subjective quality of life will be measured with the World Health Organization Quality of Life InstrumentBrief (WHOQOL-BREF) [86, 89]. Mean scores of all four domains (physical health, psychological, social relationships, environment) will be used. The revised Symptom Checklist (SCL-90-R) will be used as a reliable and valid measure to assess general psychopathology in youth $[86$, 90]. The measure consists of 90 items, which will be rated on a 5-point scale. The total sum score of the SCL-90-R will be used for analysis. We will use the 24item version of the Brief Psychiatric Rating Scale (BPRS) $[91,92]$ as a validated interviewer measure to assess clinical symptoms of psychopathology in youth [86]. All items are rated on a 7-point scale and, for the analysis, the BPRS total score will be computed.

The Trimbos Institute and Institute of Medical Technology Assessment Questionnaire for Costs associated with Psychiatric Illness (TiC-P) [93] will be used to collect data on service use (including admission to inpatient services) and cost for cost-effectiveness analysis. Last, data on health-related quality of life will be operationalized by quality-adjusted life years (QALYs), which will be calculated based on the EQ-5D 5-level version of the "EuroQoL" group (EQ-5D-5L) for cost-utility analysis [94].

\section{Process evaluation}

A process evaluation will be performed following the methodology of realist evaluation [95]. Initial program theories will be developed based on transcribed data from a focus group with stakeholders as well as expert interviews. Overarching program theory and accompanying context-mechanism-outcome configurations will be tested among intervention users (individual interviews with participants who have completed the SELFIE intervention) as well as those who deliver the intervention (focus group with SELFIE therapists), through iterative data collection. Atlas.Ti will be used as software to support the process of our analyses.

\section{Acceptability, adherence, and fidelity}

We will carefully assess acceptability, safety, adherence, and fidelity of the SELFIE intervention. Participants in the experimental condition will be asked to complete a debriefing questionnaire, which assesses acceptability, satisfaction, and whether or not there were beneficial effects of the EMI tasks and sessions. Also the Working Alliance Inventory (WAI) [96] will be completed by the 
participant and the SELFIE therapist providing the SELFIE intervention. Adherence to the intervention will be assessed using the implicit EMI adherence data recorded by the app (e.g., number and duration of completed EMI interactive, enhancing and consolidating tasks). Further, the attended face-to-face sessions will be audio recorded and adherence will be rated on a visual analog scale (ranging from $0=$ "not at all" to $11=$ "very much") by a clinical psychologist or researchers (supervised by a clinical psychologist).

\section{Other measures}

A socio-demographic schedule will be used to assess basic socio-demographic and clinical characteristics including age, gender, employment status, and level of education. Resilience will be assessed with the Temperament and Characteristics Inventory (TCI) [97]. Last, other confounders, such as alcohol and substance use (Composite International Diagnostic Interview (CIDI), sections B, J, and L) [98], medication use, treatment classification, and social support (Childhood Experience of Care and Abuse (CECA), section social support) [67], will also be assessed.

\section{Sample size}

Previous studies demonstrated that third-wave cognitive behavioral therapy (CBT) [22, 99, 100], including CBT focusing on self-esteem [51, 101], may lead to reductions in symptoms of psychopathology of moderate to large effect size. In line with previous research, the power calculation is based on the primary outcome of level of self-esteem as measured with the RSES [51]. Power simulation in the $\mathrm{R}$ environment indicated that a sample size of 130 participants (65 per condition) is sufficient to test our primary hypothesis of the effect of condition (SELFIE + TAU vs. TAU) on self-esteem, while controlling for self-esteem at baseline. Specifically, this will allow us to detect an effect size (standardized mean difference (SMD)) of 0.3 (experimental vs. control condition), i.e., a difference that is considered clinically relevant, at (at least) post-intervention or 6-month follow-up with a power of 0.87 (primary hypothesis), and, at long term, (at least) at one of the postintervention and follow-up time points (6-month, 18month, and 24-month follow-up), with a power of 0.82 when testing at alpha $=0.05$ using linear mixed modeling. Based on our previous and ongoing work, we will allow for a $25 \%$ attrition rate at 2-year follow-up, which will result in a loss to follow-up of around 22 individuals per condition on average (see Fig. 1). Hence, we will recruit a total sample of 174 participants (87 experimental, 87 control condition) at baseline.

\section{Randomization and blinding}

Each participant will be randomized at a 50:50 ratio to the experimental or control condition after completing the baseline assessment. Randomization will be conducted through a computer-generated sequence, stratified by region of a collaborating center or as external admission. The assessors will be blind to the allocation of subjects when assessing participants at postintervention, 6-month, 18-month, and 24-month followup. After random allocation to the experimental condition, the names and contact details of the participants will be passed on to the SELFIE-therapist providing the SELFIE intervention. This will be done through an independent researcher. This researcher will inform the assessors when assessments at post-intervention and follow-up need to take place for each individual participant. The design of this study is single blinded, because SELFIE therapists and patients cannot be masked towards the allocation of patients to the experimental or control condition. Any data specific to the intervention condition (e.g., on treatment fidelity) will be stored in a separate database. Any breaks in masking will be documented in the trial master file and another assessor will be allocated to complete the next set of assessments where possible.

\section{Assessment of safety}

Serious adverse events (SAE), which include any serious incidents that result in death, persistent or significant disability or incapacity, require (extension of) hospitalization or are life threatening, will be monitored and collected throughout the study period. In case of occurrence, SAEs will be reported to the accredited Medical Ethics Review Committee (MERC), the Data Monitoring and Ethics Committee (DMEC), and the Trial Steering Committee (TSC). While carefully documented, it is not expected that any SAE will occur as a result of the intervention. The DMEC will advise on any ethical or safety concerns, monitor evidence for intervention harm (e.g., SAEs) for the experimental condition, and review whether these events are in line with expectations. If deemed necessary, the DMEC can recommend to the Coordinator and TSC for interim analyses to be conducted and the trial to be terminated prematurely. All reported (serious) adverse events will be reported in publications of findings from this study.

\section{Statistical analysis}

A full statistical analysis plan will be written and published prior to unblinding of the study and before any analysis is being undertaken. The trial data set will be accessed by the investigators to test the primary hypothesis of an improvement in self-esteem at postintervention and 6-month follow-up in a priori planned 
statistical analysis when data collection for assessments at 6-month follow-up has been completed while retaining masking of assessors until the last assessment of the last participant at 24-month follow-up. We will use a linear regression model with the primary outcome of self-esteem at post-intervention and 6-month follow-up entered as the dependent variable and self-esteem measured at baseline, condition (SELFIE + TAU vs. TAU), time (as a two-level factor), center (as a four-level factor), the baseline $\times$ time interaction, and a time $\times$ condition interaction term as independent variables, in line with the intention-to-treat principle. All randomized participants will be included in the analysis and will be analyzed according to the intention to treat principle. Residuals within subjects will be allowed to be correlated with a completely unstructured variance-covariance matrix to take within-subject clustering of repeated measures into account. We will fit the model using Restricted Maximum Likelihood (REML [102]) in Stata 15 [103], which allows for all available data to be used assuming that data is missing at random if all variables associated with missing values are included in the model $[104,105]$. Therefore, potential bias due to attrition over the study period, differences between centers, or as a function of baseline self-esteem will be minimized by the model. We will make every effort to assess all participants at post-intervention and follow-up. To test the main effect of condition, an omnibus test of no difference between the two conditions at all two time points (Wald-type test with $\mathrm{df}=2$ and alpha $=.05$ ) will be used. The two time-specific contrasts will be examined if the omnibus test is statistically significant to determine at which time points significant differences are present (each tested at alpha $=.05$ ). The two time-specific contrasts (to determine at which time points significant differences are present) will only be examined if the omnibus test is significant and, hence, the family-wise type I error rate of finding at least one significant difference at the three time points is controlled at alpha $=.05$. Hypotheses in relation to secondary outcomes of momentary self-esteem, positive and negative schematic beliefs of self, resilience, emotional well-being, general psychopathology, functioning, and quality of life at postintervention and 6-month follow-up will be tested following the same steps. The investigators will access the trial data set to test hypotheses in relation to all four time points (i.e., post-intervention, 6-month, 18-month, and 24-month follow-up) in a priori planned statistical analysis when data collection for assessments at 24month follow-up has been completed. For hypotheses in relation to primary and secondary outcomes at all four time points (i.e., post-intervention, 6-month, 18-month, and 24-month follow-up), the main effect of condition will be tested using, again, an omnibus test of no difference between the two groups at all four time points (Wald-type test with $\mathrm{df}=4$ and alpha $=.05$ ). The four time-specific contrasts will be examined to determine at which time points significant differences are present (each tested at alpha $=.05$ ), if the omnibus test shows to be statistically significant. Since randomization will be performed in blocks, stratified by region of collaborating center or as external admission, all analyses will include this as a covariate, even though it is not expected this variable will lead to bias. As participants will be randomly assigned to experimental and control condition, no differences across conditions are expected in other study parameters (socio-demographics, alcohol and substance use, medication use, treatment classification, social support and self-compassion). If, however, in contrast to what would be expected, there are significant differences at baseline in any of these parameters across conditions, these will be included as covariate(s) in analyses with primary and secondary outcomes as dependent variable. As ESM data have a multilevel structure, multiple ESM observations (level 1) will be treated as nested within time points (i.e., baseline, postintervention and 6-month, 18-month, and 24-month follow-up) (level 2) and time points will be treated as nested within subjects (level 3).

Cost-effectiveness analysis (CEA) will be conducted based on service use and cost data collected using the TiC-P. Cost-utility analysis (CUA) will be conducted using quality-adjusted life years (QALYs), which will be calculated based on the EQ-5D-5L. For both CEA and CUA, the incremental cost-effectiveness ratio (ICER) will be calculated, which reflects the extra cost needed (or saved) per one unit increase in self-esteem or QALY gained, respectively.

Descriptive statistics will be used and confidence intervals constructed as appropriate to compute basic sample characteristics and summarize findings on acceptability, safety, and intervention fidelity of, as well as adherence to the intervention.

\section{Interim analyses and stopping guidelines}

Since it is not expected that any harm will occur related to participation in this study, there are no predefined stopping guidelines and no a priori planned interim analyses. The DMEC can recommend to the Coordinator and TSC for interim analyses to be conducted if deemed necessary because of any ethical or safety concerns.

\section{Research governance}

Maastricht University is the sponsor of this study. The trial has received ethical approval from the Medical Ethics Review Committee (MERC) at Maastricht University Medical Centre (MUMC), the Netherlands (reference: NL64393.068.17). Amendments to the study protocol 
will be submitted to the MERC for approval, then communicated to all relevant parties (DMEC, TSC, the sponsor, funder, and collaborating centers) and will be updated in the clinical trial registry. In case of deviations from the study protocol, a breach report form will be used for documentation. The handling of the data will be in compliance with the Dutch and European General Data Protection Regulation (GDPR). If a participant withdraws their consent, all data from that participant will be destroyed. No biological specimens will be collected in this trial. All data will be handled confidentially and will be coded using a number according the order of entry. In line with the GDPR, all data will be securely stored and personal data will be stored separately from the number-coded data. Consistent with the consortium agreement of this study, the coordinator will have overall responsibility for the trial and will be responsible for the day-to-day management of the project. The project leader advices on, and supports, the coordinator in the day-to-day management of the project. Each party (i.e., School for Mental Health and Neuroscience, Mondriaan, Levvel, Academic Medical Centre Amsterdam, Parnassia) appoints its lead scientist on the project as principal investigator (PI). The coordinator and project leader will liaise closely with all PIs on recruitment and consent procedures. The Trial Management Committee will meet monthly and includes the coordinator, the project leader and all PIs. It will be chaired by the coordinator and will manage the day-to-day running of the study, audit the trial conduct, and oversee preparation of reports to the MERC, the TSC, and the DMEC. The coordinator will permit trial-related monitoring, audits, and MERC review (conducted by the Clinical Trial Center Maastricht, which is independent from the study sponsor (i.e., Maastricht University)). The TSC will meet at least annually to provide independent overall supervision of the trial, to approve the protocol and any amendments and to monitor progress (e.g., data completion rates and adherence to the protocol). Also, the DMEC will meet at least annually. The DMEC will advise on ethical or safety concerns and, for the experimental condition, monitor evidence for intervention harm (e.g., SAEs) and review whether these events are in line with expectations. The DMEC can recommend to the coordinator and TSC to be given access to all trial data as well for interim analyses to be conducted and the trial to be terminated prematurely if deemed necessary.

\section{Discussion}

Exposure to childhood adversity may have deleterious effects on self-esteem, which, in turn, has been shown to be an important putative transdiagnostic mechanism in pathways from childhood adversity to adult psychopathology $[37,38]$ and, thus, is a promising target for early intervention. Even though self-esteem is a common target of conventional psychological interventions [51, 71, $72,101]$, current psychological help remains difficult to access for youth in real-world service delivery settings $[49,50]$, and therefore, new approaches are required. The current paper presents the study protocol of a multi-center RCT to evaluate the efficacy of an EMI (SELFIE) to improve self-esteem in youth exposed to childhood adversity. SELFIE, an intervention that extends beyond or even outside the clinical setting, has been designed to improve the accessibility and efficacy of psychological interventions for youth exposed to childhood adversity $[49,50]$. The potential effects of the SELFIE intervention may help to minimize the deleterious impact of, and hence, resilience to, childhood adversity by improving self-esteem and, thereby, prevent the development of severe and enduring mental disorder later in life and reduce disease burden. This study contains several unique and novel aspects. To our knowledge, SELFIE is the first transdiagnostic EMI that focuses on improving self-esteem in youth exposed to childhood adversity, which will inform our understanding of self-esteem as a psychological mechanism as well as the growing knowledge of mHealth intervention development and implementation, in particular for EMIs. An advantage of EMIs is that the intervention components are delivered in, and therefore more easily translated to, diverse contexts of daily life [54]. In doing so, the SELFIE intervention focuses on positive rather than negative self-esteem, that is, the goal of SELFIE is to build a competing positive self-esteem, without directly targeting more deeply rooted negative self-esteem [72]. This makes this low-level intervention suitable as a guided self-help EMI that is easily accessible, individually tailored and offered in daily life. Also, the multi-center RCT design implemented in different regions of the Netherlands will provide high external validity of findings. Cost effectiveness and cost utility will inform implementation, and the process evaluation on acceptability, treatment adherence, and treatment fidelity will provide important data on potential barriers, but also on potential facilitators for implementation.

\section{Trial status}

The trial has been registered at trialregister.nl (no. NTR 7475) in November 2018, and all study procedures were approved by the MERC at MUMC in August 2018. We are currently working with protocol version 10, originating from February 2021. Recruitment started in December 2018, the first enrollment was in January 2019, recruitment was completed in June 2021, and outcome assessment will continue until December 2022. 


\section{Abbreviations}

AE: Adverse event; BCSS: Brief Core Schema Scales; BPRS: Brief Psychiatric Rating Scale; CECA: Childhood Experience of Care and Abuse; CIDI: Composite International Diagnostic Interview; CTQ: Childhood Trauma Questionnaire; DMEC: Data Monitoring and Ethics Committee; EMI: Ecological Momentary Intervention; ESM: Experience Sampling Method; EQ-5D-

5L: EuroQol - 5 Dimensions - 5 Level version; K10: Kessler Psychological Distress Scale; MERC: Medical Ethics Review Committee; MUMC: Maastricht University Medical Centre; mHealth: Mobile Health; PANAS: Positive and Negative Affect Scale; RCT: Randomized controlled trial; RBQ: Retrospective Bullying Questionnaire; RSES: Rosenberg Self-Esteem Scale; SELFIE: Ecological momentary intervention for improving self-esteem; SERS: Self-Esteem Rating Scale; SOFAS: Social and Occupational Functioning Assessment Scale; TAU: Treatment as usual; TCl: Temperament and Characteristic Inventory; TiCP: Trimbos Institute and Institute of Medical Technology Assessment Questionnaire for Costs associated with Psychiatric Illness; TSC: Trial Steering Committee; WHOQOL-BREF: World Health Organization Quality of Life Instrument-Brief

\section{Acknowledgements}

We are indebted to all individuals, who participate in the SELFIE study and are essential for its successful completion. We thank all SELFIE therapists, who offer the SELFIE intervention, researchers (Nele Volbragt, Nele Soons, Fatima Ahouch, Sanne Visser, Josefien Breedvelt, Marieke van der Pluijm, Alisa Bogdanova), and interns. Further, we are grateful to all members of the TSC (Inez Germeys, Wolfgang Viechtbauer, and Johan Lataster) and DMEC (Philippe Delespaul, Karel Borkelmans, Marjan Drukker and Viviane Thewissen), Karel Borkelmans for implementing the SELFIE intervention and ESM in PsyMate, and all clinicians who referred their clients to us.

\section{Sponsor}

Maastricht University is the primary sponsor of this study.

\section{Role of sponsors and funders}

The sponsor and funding agency do not have any role in the trial design, collection, analysis, or interpretation of data nor in writing the manuscript or in the decision to submit the report for publication.

\section{Authors' contributions}

$M D$ and UR drafted this manuscript. UR, TVA, MRP, MD, RL, DN, KS, $I H$, and MvdG contributed to the design of the study. UR is the coordinator and has managerial responsibility for the successful completion of the study. WV is the trial statistician. PD programed the intervention. MRP and IH provided the supervision and intervision for SELFIE therapists delivering the intervention. UR, TvA, RL, DN, MvdG, RL, CB, and DvdB are the Pls and, with $M D, M R P, I H$, and JB, are all involved in the recruitment of participants and data collection. All authors were involved in writing and have read and approved the final manuscript. Established guidelines for authorship (https:// www.bmj.com/aboutbmj/resources-authors/article-submission/ authorshipcontributorship) will be followed for authorship eligibility in future publications from this study. It is not intended to use professional writers.

\section{Funding}

This work was supported by a ZonMw mental health research program grant (dossier number 60-63600-98-346) and a DFG Heisenberg professorship (no. 389624707) to UR.

\section{Availability of data and materials}

The data resulting from the current study will not be publicly available. The data, protocol, statistical code, and a list of study sites are available from the corresponding author on reasonable request.

\section{Declarations}

\section{Ethics approval and consent to participate}

The trial has received ethical approval from the Medical Ethics Review Committee (MERC) at Mastricht University Medical Centre (MUMC), the Netherlands (reference: NL64393.068.17) at August 1, 2018. Written informed consent will be obtained from all participants before inclusion in the study. A translated version of the informed consent document can be found in the supplementary materials. The Clinical Trial Center Maastricht, which is independent from the study sponsor (i.e., Maastricht University), will conduct trial-related monitoring, audits and MERC review. The sponsor has an insurance that covers for damage to the research participants though injury or death caused by the study that has become apparent during the study or within four years after the termination of the study.

\section{Competing interests}

The authors declare to have no competing interests.

\section{Author details}

1Department of Psychiatry and Neuropsychology, School for Mental Health and Neuroscience, Maastricht University, Maastricht, The Netherlands. ${ }^{2}$ Department of Public Mental Health, Central Institute of Mental Health, Medical Faculty Mannheim, University of Heidelberg, Mannheim, Germany. ${ }^{3}$ Mondriaan, Maastricht, The Netherlands. ${ }^{4}$ Department of Child and Adolescent Psychiatry, Amsterdam UMC, location Academic Medical Center, Amsterdam, The Netherlands. ${ }^{5}$ Levvel, academic centre for child and adolescent psychiatry, Amsterdam, The Netherlands. ${ }^{6}$ Prodeba Mental Health Care, Leiden, The Netherlands. 'Department of Psychiatry, Amsterdam University Medical Centers (location AMC), Amsterdam, The Netherlands. ${ }^{8}$ Centre for Urban Mental Health, University of Amsterdam, Amsterdam, The Netherlands. ${ }^{9}$ Department of Clinical Psychology, VU University, Amsterdam, The Netherlands. ${ }^{10}$ Parnassia Academy, The Hague, The Netherlands. ${ }^{11}$ Department of Health Psychology, Faculty of Psychology, University of Leuven, Leuven, Belgium. ${ }^{12}$ Centre for Epidemiology and Public Health, Health Service and Population Research Department, Institute of Psychiatry, Psychology \& Neuroscience, King's College London, London, UK.

Received: 9 April 2021 Accepted: 1 September 2021 Published online: 19 September 2021

\section{References}

1. Copeland W, Shanahan L, Costello EJ, Angold A. Cumulative prevalence of psychiatric disorders by young adulthood: a prospective cohort analysis from the Great Smoky Mountains Study. J Am Acad Child Adolesc Psychiatry. 2011;50(3):252-61. https://doi.org/10.1016/j.jaac.2010.12.014.

2. Kessler RC, Berglund P, Demler O, Jin R, Merikangas KR, Walters EE. Lifetime prevalence and age-of-onset distributions of DSM-IV disorders in the National Comorbidity Survey Replication. Arch Gen Psychiatry. 2005;62(6): 593-602. https://doi.org/10.1001/archpsyc.62.6.593.

3. Kim-Cohen J, Caspi A, Moffitt TE, Harrington H, Milne BJ, Poulton R. Prior juvenile diagnoses in adults with mental disorder: developmental followback of a prospective-longitudinal cohort. Arch Gen Psychiatry. 2003;60(7): 709-17. https://doi.org/10.1001/archpsyc.60.7.709.

4. Harhay $\mathrm{MO}$, King $\mathrm{CH}$. Global burden of disease in young people aged 10-24 years. Lancet. 2012;379(9810):27-8. https://doi.org/10.1016/S0140-6736(12 60019-1.

5. Gore FM, Bloem PJ, Patton GC, Ferguson J, Joseph V, Coffey C, et al. Global burden of disease in young people aged 10-24 years: a systematic analysis. Lancet. 2011;377(9783):2093-102. https://doi.org/10.1016/S0140-6736(11 )60512-6.

6. de Girolamo G, Dagani J, Purcell R, Cocchi A, McGorry PD. Age of onset of mental disorders and use of mental health services: needs, opportunities and obstacles. Epidemiol Psychiatr Sci. 2012;21(1):47-57. https://doi.org/10.1 017/s2045796011000746.

7. Mangalore R, Knapp M. Cost of schizophrenia in England. J Ment Health Policy Econ. 2007;10(1):23-41.

8. McCrone P, Dhanasiri S, Patel A, Knapp M, Lawton-Smith M. Paying the price - the cost of mental health care in England to 2026. London; 2008

9. The Schizophrenia Commission. The abandoned illness: a report by the Schizophrenia Commission. London; 2012.

10. Wood D, Crapnell T, Lau L, Bennett A, Lotstein D, Ferris M, et al. Emerging Adulthood as a Critical Stage in the Life Course. In: Halfon N, Forrest CB, Lerner RM, Faustman EM, editors. Handbook of Life Course Health Development. Cham (CH): Springer; 2018. p. 123-43. https://doi.org/10.1 007/978-3-319-47143-3_7.

11. McGorry PD, Purcell R, Hickie IB, Jorm AF. Investing in youth mental health is a best buy. Medical Journal of Australia. 2007;187(S7):S5-7. https://doi. org/10.5694/j.1326-5377.2007.tb01326.x. 
12. McGorry P, Van Os J. Redeeming diagnosis in psychiatry: timing versus specificity. The Lancet. 2013;381(9863):343-5. https://doi.org/10.1016/S01406736(12)61268-9.

13. Ruggero CJ, Kotov R, Hopwood CJ, First M, Clark LA, Skodol AE, et al. Integrating the Hierarchical Taxonomy of Psychopathology (HiTOP) into clinical practice. J Consult Clin Psychol. 2019;87(12):1069-84. https://doi. org/10.1037/ccp0000452.

14. Kotov R, Krueger RF, Watson D, Achenbach TM, Althoff RR, Bagby RM, et al. The Hierarchical Taxonomy of Psychopathology (HiTOP): A dimensional alternative to traditional nosologies. J Abnorm Psychol. 2017;126(4):454-77. https://doi.org/10.1037/abn0000258

15. van Os J. The dynamics of subthreshold psychopathology: implications for diagnosis and treatment. Am J Psychiatry. 2013;170(7):695-8. https://doi. org/10.1176/appi.ajp.2013.13040474

16. van Os J, Reininghaus U. Psychosis as a transdiagnostic and extended phenotype in the general population. World Psychiatry. 2016;15(2):118-24. https://doi.org/10.1002/wps.20310.

17. Holmes EA, O'Connor RC, Perry VH, Tracey I, Wessely S, Arseneault L, et al. Multidisciplinary research priorities for the COVID-19 pandemic: a call for action for mental health science. Lancet Psychiatry. 2020;7(6):547-60. https://doi.org/10.1016/s2215-0366(20)30168-1.

18. Rauschenberg C, Schick A, Goetzl C, Roehr S, Riedel-Heller SG, Koppe G, et al. Social isolation, mental health and use of digital interventions in youth during the COVID-19 pandemic: a nationally representative survey. 2020 DOI: https://doi.org/10.31234/osf.io/v64hf.

19. Loades ME, Chatburn E, Higson-Sweeney N, Reynolds S, Shafran R, Brigden $A$, et al. Rapid systematic review: the impact of social isolation and loneliness on the mental health of children and adolescents in the context of COVID-19. J Am Acad Child Adolesc Psychiatry. 2020;59(11):1218-1239.e3. https://doi.org/10.1016/j.jaac.2020.05.009.

20. Rauschenberg C, Schulte-Strathaus J, van Os J, Goedhart M, Schieveld J, Reininghaus U. Life events and stress sensitivity in youth's daily life: an ecological momentary assessment study. [Unpublished manuscript]. Department of Public Mental Health, University of Heidelberg. 2020.

21. Schick A, Paetzold I, Rauschenberg C, Hirjak D, Deuschle M, Grimmer Y, et al. Efficacy of a novel, accessible, transdiagnostic, compassion-focused ecological momentary intervention for enhancing resilience in help-seeking youth (EMlcompass). PsychArchives [Internet]. 2020.

22. Rauschenberg C, Böcking B, Pätzold I, Deckers D, Schruers K, Heunen I, et al. An ecological momentary compassion-focused intervention for enhancing resilience in help-seeking youths: a pilot study. PsyArXiv. 2020. DOI: https:// doi.org/10.31234/osf.io/txhp7.

23. Chen LP, Murad MH, Paras ML, Colbenson KM, Sattler AL, Goranson EN, et al. Sexual abuse and lifetime diagnosis of psychiatric disorders: systematic review and meta-analysis. Mayo Clin Proc. 2010;85(7):618-29. https://doi. org/10.4065/mcp.2009.0583.

24. Frissen $A$, Lieverse $R$, Drukker $M$, van Winkel $R$, Delespaul $P$, Investigators $G$. Childhood trauma and childhood urbanicity in relation to psychotic disorder. Soc Psychiatry Psychiatr Epidemiol. 2015;50(10):1481-8. https://doi. org/10.1007/s00127-015-1049-7.

25. Matheson SL, Shepherd AM, Pinchbeck RM, Laurens KR, Carr VJ. Childhood adversity in schizophrenia: a systematic meta-analysis. Psychol Med. 2013; 43(2):225-38. https://doi.org/10.1017/S0033291712000785.

26. Varese F, Smeets F, Drukker M, Lieverse R, Lataster T, Viechtbauer W, et al. Childhood adversities increase the risk of psychosis: a meta-analysis of patient-control, prospective- and cross-sectional cohort studies. Schizophr Bull. 2012;38(4):661-71. https://doi.org/10.1093/schbul/sbs050.

27. Kraan T, van Dam DS, Velthorst E, de Ruigh EL, Nieman DH, Durston S, et al. Childhood trauma and clinical outcome in patients at ultra-high risk of transition to psychosis. Schizophr Res. 2015;169(1-3):193-8. https://doi.org/1 0.1016/j.schres.2015.10.030

28. Kraan T, Velthorst E, Smit F, de Haan L, van der Gaag M. Trauma and recent life events in individuals at ultra high risk for psychosis: review and meta-analysis. Schizophr Res. 2015;161(2-3):143-9. https:/doi.org/10.1016/j.schres.2014.11.026.

29. Kraan TC, Ising HK, Fokkema M, Velthorst E, van den Berg DP, Kerkhoven M, et al. The effect of childhood adversity on 4-year outcome in individuals at ultra high risk for psychosis in the Dutch Early Detection Intervention Evaluation (EDIE-NL) Trial. Psychiatry Res. 2017;247:55-62. https://doi.org/1 0.1016/j.psychres.2016.11.014.

30. Rauschenberg C, van Os J, Cremers D, Goedhart M, Schieveld JNM, Reininghaus U. Stress sensitivity as a putative mechanism linking childhood trauma and psychopathology in youth's daily life. Acta Psychiatr Scand. 2017;136(4):373-88. https://doi.org/10.1111/acps.12775.

31. Reininghaus U, Gayer-Anderson C, Valmaggia L, Kempton MJ, Calem M, Onyejiaka A, et al. Psychological processes underlying the association between childhood trauma and psychosis in daily life: an experience sampling study. Psychol Med. 2016;46(13):2799-813. https://doi.org/10.1017/ s003329171600146x.

32. Kessler RC, McLaughlin KA, Green JG, Gruber MJ, Sampson NA, Zaslavsky AM, et al. Childhood adversities and adult psychopathology in the WHO World Mental Health Surveys. The British journal of psychiatry : the journal of mental science. 2010;197(5):378-85. https://doi.org/10.1192/bjp.bp.110. 080499.

33. Green JG, McLaughlin KA, Berglund PA, Gruber MJ, Sampson NA, Zaslavsky AM, et al. Childhood adversities and adult psychiatric disorders in the national comorbidity survey replication I: associations with first onset of DSM-IV disorders. Arch Gen Psychiatry. 2010;67(2):113-23. https://doi.org/1 0.1001/archgenpsychiatry.2009.186.

34. Riem MME, Karreman A. Childhood adversity and adult health: the role of developmental timing and associations with accelerated aging. Child Maltreat. 2019;24(1):17-25. https://doi.org/10.1177/1077559518795058.

35. Kirkbride JB, Jones PB. The prevention of schizophrenia--what can we learn from eco-epidemiology? Schizophr Bull. 2011;37(2):262-71. https://doi.org/1 $0.1093 /$ schbul/sbq120.

36. Reininghaus U, Morgan C, Fearon P, Hutchinson G, Morgan K, Dazzan P, et al. Modelling the interplay between childhood and adult adversity in pathways to psychosis: initial evidence from the AESOP study. Psychol Med. 2014:44(2):407-19. https://doi.org/10.1017/S0033291713000767.

37. Brown GW, Craig TK, Harris TO, Handley RV. Parental maltreatment and adulthood cohabiting partnerships: a life-course study of adult chronic depression--4. J Affect Disord. 2008;110(1-2):115-25. https://doi.org/10.1016/ j.jad.2008.01.015

38. Garety PA, Bebbington P, Fowler D, Freeman D, Kuipers E. Implications for neurobiological research of cognitive models of psychosis: a theoretical paper. Psychol Med. 2007;37(10):1377-91. https://doi.org/10.1017/s0033291 70700013x.

39. Zeigler-Hill V. The connections between self-esteem and psychopathology. Contemp Psychother. 2011;41(3):157-64. https://doi.org/10.1007/s10879-0109167-8.

40. Banyard VL, Williams LM, Siegel JA. The long-term mental health consequences of child sexual abuse: an exploratory study of the impact of multiple traumas in a sample of women. J Trauma Stress. 2001;14(4):697715. https://doi.org/10.1023/a:1013085904337.

41. Kamsner S, McCabe MP. The relationship between adult psychological adjustment and childhood sexual abuse, childhood physical abuse, and family-of-origin characteristics. J Interpers Violence. 2000;15(12):1243-61. https://doi.org/10.1177/088626000015012001.

42. Murthi M, Espelage DL. Childhood sexual abuse, social support, and psychological outcomes: a loss framework. Child Abuse Negl. 2005;29(11): 1215-31. https://doi.org/10.1016/j.chiabu.2005.03.008.

43. Mannarino AP, Cohen JA. Abuse-related attributions and perceptions, general attributions, and locus of control in sexually abused girls. J Interpers Violence. 1996;11(2):162-80. https://doi.org/10.1177/088626096011002002.

44. Fisher HL, Appiah-Kusi E, Grant C. Anxiety and negative self-schemas mediate the association between childhood maltreatment and paranoia. Psychiatry Res. 2012;196(2-3):323-4. https://doi.org/10.1016/j.psychres.2011.12.004.

45. Fisher HL, Schreier A, Zammit S, Maughan B, Munafo MR, Lewis G, et al. Pathways between childhood victimization and psychosis-like symptoms in the ALSPAC birth cohort. Schizophr Bull. 2013;39(5):1045-55. https://doi. org/10.1093/schbul/sbs088.

46. Postma MR, van Amelsvoort T, Myin-Germeys I, Gayer-Anderson C, Kempton MJ, Valmaggia L, et al. Across the continuum: fluctuations in momentary self-esteem in early psychosis [Unpublished manuscript] Psychiatry and Neuropsychology, Maastricht University; 2020

47. Daemen M, van Amelsvoort, T., Group Investigators, Reininghaus, U. Selfesteem and psychosis in daily life: an experience sampling study [Unpublished manuscript]. Psychiatry and Neuropsychology, Maastricht University. 2020

48. Overholser JC, Adams DM, Lehnert KL, Brinkman DC. Self-esteem deficits and suicidal tendencies among adolescents. J Am Acad Child Adolesc Psychiatry. 1995;34(7):919-28. https://doi.org/10.1097/00004583-19950700000016. 
49. Malla A, lyer S, McGorry P, Cannon M, Coughlan H, Singh S, et al. From early intervention in psychosis to youth mental health reform: a review of the evolution and transformation of mental health services for young people. Soc Psychiatry Psychiatr Epidemiol. 2016;51(3):319-26. https://doi.org/10.1 007/s00127-015-1165-4.

50. McGorry P, Bates T, Birchwood M. Designing youth mental health services for the 21st century: examples from Australia, Ireland and the UK. Br J Psychiatry Suppl. 2013;54(s54):s30-5. https://doi.org/10.1192/bjp.bp.112.11 9214.

51. Staring $A B$, van den Berg DP, Cath DC, Schoorl M, Engelhard IM, Korrelboom CW. Self-esteem treatment in anxiety: a randomized controlled crossover trial of Eye Movement Desensitization and Reprocessing (EMDR) versus Competitive Memory Training (COMET) in patients with anxiety disorders. Behav Res Ther. 2016;82:11-20. https://doi.org/10.1016/j.brat.2016. 04.002.

52. Reininghaus $U$, Depp CA, Myin-Germeys I. Ecological interventionist causal models in psychosis: targeting psychological mechanisms in daily life. Schizophr Bull. 2016;42(2):264-9. https://doi.org/10.1093/schbul/sbv193.

53. Myin-Germeys I, Klippel A, Steinhart H, Reininghaus U. Ecological momentary interventions in psychiatry. Curr Opin Psychiatry. 2016;29(4): 258-63. https://doi.org/10.1097/YCO.0000000000000255.

54. Heron KE, Smyth JM. Ecological momentary interventions: incorporating mobile technology into psychosocial and health behaviour treatments. Br J Health Psychol. 2010;15(Pt 1):1-39. https://doi.org/10.1348/135910709x4 66063.

55. Dinesen B, Nonnecke B, Lindeman D, Toft E, Kidholm K, Jethwani K, et al. Personalized telehealth in the future: a global research agenda. J Med Internet Res. 2016;18(3):e53. https://doi.org/10.2196/jmir.5257.

56. Reininghaus $U$. Ecological momentary interventions in psychiatry: the momentum for change in daily social context. Psychiatr Prax. 2018;45(2):5961. https://doi.org/10.1055/s-0044-101986.

57. Bidargaddi N, Schrader G, Klasnja P, Licinio J, Murphy S. Designing m-Health interventions for precision mental health support. Transl Psychiatry. 2020; 10(1):222. https://doi.org/10.1038/s41398-020-00895-2.

58. Nahum-Shani I, Smith SN, Spring BJ, Collins LM, Witkiewitz K, Tewari A, et al. Just-in-time adaptive interventions (JITAIs) in mobile health: key components and design principles for ongoing health behavior support. Ann Behav Med. 2018;52(6):446-62. https://doi.org/10.1007/s12160-016-983 0-8.

59. Steinhart H, Myin-Germeys I, Reininghaus U. Translating treatment of mental health problems to daily life: a guide to the development of ecological momentary interventions. In: Palmier-Claus J, Haddock G, Varese F, editors. Novel uses of experience sampling in mental health research. London: Routledge. in press.

60. Bakker D, Kazantzis N, Rickwood D, Rickard N. Mental health smartphone apps: review and evidence-based recommendations for future developments. JMIR mental health. 2016;3(1):e7. https://doi.org/10.2196/ mental.4984.

61. Weisel KK, Fuhrmann LM, Berking M, Baumeister H, Cuijpers P, Ebert DD. Standalone smartphone apps for mental health — a systematic review and meta-analysis. NPJ digital medicine. 2019;2(1):1-10. https://doi.org/10.1038/ s41746-019-0188-8.

62. Reininghaus $U$, Klippel A, Steinhart $H$, Vaessen $T$, Van Nierop M, Viechtbauer W, et al. Efficacy of Acceptance and Commitment Therapy in Daily Life (ACT-DL) in early psychosis: study protocol for a multi-centre randomized controlled trial. Trials. 2019;20(1):1-12.

63. Bernstein DP, Fink L. Childhood Trauma Questionnaire: a retrospective selfreport manual. San Antonio (Texas): The Psychological Corporation; 1998.

64. Scher CD, Stein MB, Asmundson GJ, McCreary DR, Forde DR. The childhood trauma questionnaire in a community sample: psychometric properties and normative data. J Trauma Stress. 2001;14(4):843-57. https://doi.org/10.1 023/A:1013058625719.

65. Wright KD, Asmundson GJ, McCreary DR, Scher C, Hami S, Stein MB. Factorial validity of the Childhood Trauma Questionnaire in men and women. Depress Anxiety. 2001;13(4):179-83. https://doi.org/10.1002/da.1034.

66. Schäfer M, Korn S, Smith PK, Hunter SC, Mora-Merchán JA, Singer MM, et al. Lonely in the crowd: recollections of bullying. Br J Dev Psychol. 2004;22(3): 379-94. https://doi.org/10.1348/0261510041552756.

67. Bifulco A, Brown GW, Harris TO. Childhood Experience of Care and Abuse (CECA): a retrospective interview measure. J Child Psychol Psychiatry. 1994; 35(8):1419-35. https://doi.org/10.1111/j.1469-7610.1994.tb01284.x.
68. Schmitt P, Allik J. Simultaneous administration of the Rosenberg self-esteem scale in 53 nations: exploring the universal and culture specific features of global self-esteem. J Pers Soc Psychol. 2009;89(4):623-42. https://doi.org/1 0.1037/0022-3514.89.4.623.

69. Rosenberg M. Society and the adolescent self-image. 10th ed. Princeton, NJ: Princeton University Press; 1965. https://doi.org/10.1515/9781400876136.

70. Korrelboom K, van der Weele K, Gjaltema M, Hoogstraten C. Competitive memory training for treating low self-esteem: a pilot study in a routine clinical setting. Behav Ther. 2009

71. Griffioen BT, van der Vegt AA, de Groot IW, de Jongh A. The effect of EMDR and CBT on low self-esteem in a general psychiatric population: a randomized controlled trial. Front Psychol. 2017:8:1910. https://doi.org/10.33 89/fpsyg.2017.01910.

72. De Neef M. Build Your Confidence with CBT: 6 Simple Steps to be Happier, More Successful, and Fulfilled: Open University Press; 2016.

73. Postma MR, Hoes I, Daemen M, van Amersvoort T, Reininghaus U. SELFIE, a transdiagnostic ecological momentary intervention for improving selfesteem in youth exposed to childhood adversity - structure of the intervention. Maastricht: Maastricht University; 2019.

74. Reininghaus U, Kempton MJ, Valmaggia L, Craig TK, Garety P, Onyejiaka A, et al. Stress sensitivity, aberrant salience, and threat anticipation in early psychosis: an experience sampling study. Schizophr Bull. 2016;42(3):712-22. https://doi.org/10.1093/schbul/sbv190.

75. Myin-Germeys I, Birchwood M, Kwapil T. From environment to therapy in psychosis: a real-world momentary assessment approach. Schizophr Bull. 2011;37(2):244-7. https://doi.org/10.1093/schbul/sbq164.

76. Myin-Germeys I, Oorschot M, Collip D, Lataster J, Delespaul P, van Os J. Experience sampling research in psychopathology: opening the black box of daily life. Psychol Med. 2009;39(9):1533-47. https://doi.org/10.1017/ S0033291708004947.

77. Everaert J, Koster EHW, Schacht R, De Raedt R. Evaluatie van de psychometrische eigenschappen van de Rosenberg zelfwaardeschaal in een poliklinische psychiatrische populatie. Gedragstherapie. 2010;43:307-17.

78. Thewissen V, Bentall RP, Lecomte T, van Os J, Myin-Germeys I. Fluctuations in self-esteem and paranoia in the context of daily life. J Abnorm Psychol. 2008;117(1):143-53. https://doi.org/10.1037/0021-843x.117.1.143.

79. Thewissen V, Bentall RP, Oorschot M. J AC, van Lierop T, van Os J, et al. Emotions, self-esteem, and paranoid episodes: an experience sampling study. Br J Clin Psychol. 2011;50(2):178-95. https://doi.org/10.1348/01446651 0X508677.

80. Lecomte T, Corbiere M, Laisne F. Investigating self-esteem in individuals with schizophrenia: relevance of the Self-Esteem Rating Scale-Short Form. Psychiatry Res. 2006;143(1):99-108. https://doi.org/10.1016/j.psychres.2005. 08.019.

81. Vaessen T, Viechtbauer W, van der Steen Y, Gayer-Anderson C, Kempton MJ, Valmaggia $L$, et al. Recovery from daily-life stressors in early and chronic psychosis. Schizophr Res. 2019;213:32-9. https://doi.org/10.1016/j.schres.201 9.03.011.

82. Watson D, Clark LA, Tellegen A. Development and validation of brief measures of positive and negative affect: the PANAS scales. J Pers Soc Psychol. 1988;54(6):1063-70. https://doi.org/10.1037/0022-3514.54.6.1063.

83. Myin-Germeys I, van Os J, Schwartz JE, Stone AA, Delespaul PA. Emotional reactivity to daily life stress in psychosis. Arch Gen Psychiatry. 2001;58(12): 1137-44. https://doi.org/10.1001/archpsyc.58.12.1137.

84. Fowler D, Freeman D, Smith B, Kuipers E, Bebbington $P$, Bashforth $H$, et al. The Brief Core Schema Scales (BCSS): psychometric properties and associations with paranoia and grandiosity in non-clinical and psychosis samples. Psychol Med. 2006;36(6):749-59. https://doi.org/10.1017/S0033291 706007355.

85. Kessler RC, Andrews G, Colpe LJ, Hiripi E, Mroczek DK, Normand SL, et al. Short screening scales to monitor population prevalences and trends in non-specific psychological distress. Psychol Med. 2002;32(6):959-76. https:// doi.org/10.1017/S0033291702006074.

86. Kwan B, Rickwood DJ. A systematic review of mental health outcome measures for young people aged 12 to 25 years. BMC Psychiatry. 2015;15(1): 279. https://doi.org/10.1186/s12888-015-0664-x.

87. Goldman HH, Skodol AE, Lave TR. Revising axis V for DSM-IV: a review of measures of social functioning. Am J Psychiatry. 1992;149(9):1148-56. https://doi.org/10.1176/ajp.149.9.1148.

88. Birchwood M, Smith J, Cochrane R, Wetton S, Copestake S. The Social Functioning Scale. The development and validation of a new scale of social 
adjustment for use in family intervention programmes with schizophrenic patients. Br J Psychiatry. 1990;157(6):853-9. https://doi.org/10.1192/bjp.157.6. 853.

89. Whogol Group. Development of the World Health Organization WHOQOLBREF quality of life assessment. Psychol Med. 1998;28(3):551-8. https://doi. org/10.1017/S0033291798006667.

90. Derogatis LR. SCL-90-R, administration, scoring \& procedures manual-I for the R(evised) version. Baltimore, MD: Johns Hopkins University School of Medicine; 1977.

91. Lukoff D, Liberman RP, Nuechterlein KH. Symptom monitoring in the rehabilitation of schizophrenic patients. Schizophr Bull. 1986;12(4):578-602. https://doi.org/10.1093/schbul/12.4.578.

92. Overall JE, Hollister LE, Pichot P. Major psychiatric disorders. A fourdimensional model. Arch Gen Psychiatry. 1967;16(2):146-51. https://doi. org/10.1001/archpsyc.1967.01730200014003.

93. Bouwmans C, De Jong K, Timman R, Zijlstra-Vlasveld M, Van der FeltzCornelis C, Tan Swan S, et al. Feasibility, reliability and validity of a questionnaire on healthcare consumption and productivity loss in patients with a psychiatric disorder (TiC-P). BMC Health Serv Res. 2013;13(1):217. https://doi.org/10.1186/1472-6963-13-217.

94. Herdman M, Gudex C, Lloyd A, Janssen M, Kind P, Parkin D, et al. Development and preliminary testing of the new five-level version of EQ-5D (EQ-5D-5L). Quality of life research : an international journal of quality of life aspects of treatment, care and rehabilitation. 2011;20(10):1727-36. https:// doi.org/10.1007/s11136-011-9903-x.

95. Pawson R, Tilley N. An introduction to scientific realist evaluation. Evaluation for the 21st century: A handbook. 1997.1997:405-18.

96. Horvath AO, Greenberg LS. Development and validation of the Working Alliance Inventory. J Couns Psychol. 1989;36(2):223-33. https://doi.org/10.1 037/0022-0167.36.2.223.

97. Cloninger CR, Przybeck TR, Svrakic DM, Wetzel RD. The Temperament and Character Inventory (TCI): a guide to its development and use; 1994.

98. Organization WH. Composite international diagnostic interview (CIDI), Version 1.0. Geneva, Switzerland: World Health Organization; 1990

99. Hunot V, Moore TH, Caldwell DM, Furukawa TA, Davies P, Jones H, et al. Third wave' cognitive and behavioural therapies versus other psychological therapies for depression. Cochrane Database Syst Rev. 2013;10. https://doi. org/10.1002/14651858.CD008704.pub2.

100. Johns LC, Oliver JE, Khondoker M, Byrne M, Jolley S, Wykes T, et al. The feasibility and acceptability of a brief Acceptance and Commitment Therapy (ACT) group intervention for people with psychosis: the 'ACT for life'study. J Behav Ther Exp Psychiatry. 2016;50:257-63. https://doi.org/10.1016/j.jbtep.2 015.10.001.

101. Staring T. Self-Esteem Treatment in Anxiety (SETA) trial. GZ-Psychologie. 2014;6(5):10-2. https://doi.org/10.1007/s41480-014-0040-3.

102. Richardson AM, Welsh AH. Robust restricted maximum likelihood in mixed linear models. Biometrics. 1995;51(4):1429-39. https://doi.org/10.2307/25332 73.

103. StataCorp L. Stata statistical software: Release 15 (2017). StataCorp LP: College Station, TX; 2017.

104. StataCorp L. Stata survival analysis reference manual; 2017.

105. Little RJ, Rubin DB. Statistical analysis with missing data: John Wiley \& Sons; 2019. https://doi.org/10.1002/9781119482260.

\section{Publisher's Note}

Springer Nature remains neutral with regard to jurisdictional claims in published maps and institutional affiliations.

Ready to submit your research? Choose BMC and benefit from:

- fast, convenient online submission

- thorough peer review by experienced researchers in your field

- rapid publication on acceptance

- support for research data, including large and complex data types

- gold Open Access which fosters wider collaboration and increased citations

- maximum visibility for your research: over $100 \mathrm{M}$ website views per year

At BMC, research is always in progress.

Learn more biomedcentral.com/submissions 